\title{
SIMULATION OF IONIZATION EFFECTS FOR HIGH-DENSITY POSITRON DRIVERS IN FUTURE PLASMA WAKEFIELD EXPERIMENTS*
}

\author{
D.L. Bruhwiler, D.A. Dimitrov and J.R. Cary", Tech-X Corp., Boulder, CO 80301, USA \\ E. Esarey, W.P. Leemans, LBNL, Berkeley, CA 94720, USA
}

\begin{abstract}
The plasma wakefield accelerator (PWFA) concept has been proposed [1] as a potential energy doubler for present or future electron-positron colliders. Recent particle-in-cell (PIC) simulations have shown [2] that the self-fields of the required electron beam driver can tunnel ionize neutral $\mathrm{Li}$, leading to plasma wake dynamics differing significantly from that of a preionized plasma. It has also been shown [3], for the case of a preionized plasma, that the plasma wake of a positron driver differs strongly from that of an electron driver. We will present new PIC simulations, using the OOPIC [4] code, showing the effects of tunneling ionization on the plasma wake generated by high-density positron drivers. The results will be compared to previous work on electron drivers with tunneling ionization and positron drivers without ionization. Parameters relevant to the energy doubler and the upcoming E-164x experiment [5] at the Stanford Linear Accelerator Center will be considered.
\end{abstract}

\section{INTRODUCTION}

Plasma-based accelerators have demonstrated accelerating fields in excess of $100 \mathrm{GV} / \mathrm{m}$ (for a review, see Ref. [6]), which is two to three orders beyond that of conventional technology. The acceleration field in a PWFA $[7,8]$ is generated by a relativistic electron or positron beam. The self-fields of the drive bunch excite a wakefield that propagates close to the speed of light, $c$.

We present 2-D OOPIC simulations of PWFA configurations for both e+ and e- drive beams. Fieldinduced tunneling ionization is an important effect that has to be considered when designing advanced accelerators. The electric field magnitudes that are reached can be sufficient to tunnel ionize certain neutral gases. The released electrons contribute to the plasma density and affect the physics of the propagating beam and the formed wake.

Initially, we show results that demonstrate agreement with results previously published by Lee et al. [3]. Then we show new simulations for positron and electron beams with expected E-164x parameters. We studied both e+ and e- beams propagating in preionized plasma and in Cs neutral gas with the same density as in the preionized plasma case. The plasma is created completely by tunnelling ionization for the runs with Cs. The results for the positron beams with tunnelling ionization are the first such PIC simulations. Our parameter studies from these

\footnotetext{
*Funded by US DoE under grant no.'s DE-FG03-02ER83557, DE-

FG02-01ER41178, DE-FG03-95ER40926 \& DE-AC03-76SF00098.

\#also University of Colorado, Boulder, CO 80309, USA
}

simulations are summarized for both beams and are relevant to the design of the E164x experiments.

\section{COMPARISON WITH PREVIOUS WORK}

Recently, Lee et al. [3] published results from 3-D PIC simulations on plasma wakefield acceleration of a positron beam. We repeated these simulations, using 2-D cylindrical geometry, for both the electron and positron beams. Some of the relevant parameters for these benchmarking simulations are summarized in Table 1.

Table 1: Simulation Parameters

\begin{tabular}{|c|c|c|}
\hline Quantity & Benchmarking & E-164x \\
\hline $\mathrm{dt}[\mathrm{s}]$ & $1-2 \times 10^{-14}$ & $1.5 \times 10^{-15}$ \\
\hline $\mathrm{dz}[\mu \mathrm{m}]$ & $12.75-25$ & 1 \\
\hline $\mathrm{dr}[\mu \mathrm{m}]$ & $6.35-12.7$ & 1 \\
\hline $\mathrm{n}_{\mathrm{e}}\left[\mathrm{cm}^{-3}\right]$ & $4.3 \times 10^{14}$ & $10^{16}-10^{17}$ \\
\hline $\mathrm{n}_{\mathrm{Cs}}\left[\mathrm{cm}^{-3}\right]$ & -- & $10^{16}-10^{17}$ \\
\hline$\sigma_{\mathrm{r}}[\mu \mathrm{m}]$ & 75 & 20 \\
\hline$\sigma_{\mathrm{z}}[\mu \mathrm{m}]$ & 400 & 20 \\
\hline $\mathrm{N}_{\mathrm{ptcls}}$ & $2 \times 10^{10}$ & $1 \times 10^{10}$ \\
\hline $\mathrm{E}_{\text {beam }}[\mathrm{GeV}]$ & 30 & 30 \\
\hline
\end{tabular}

In our OOPIC simulations, a positron or an electron beam with Gaussian distributions along the longitudinal and transverse directions is propagated in preionized plasma for $2 \mathrm{~cm}$. Figure 1 shows a surface plot of the longitudinal electric field $\mathrm{E}_{\mathrm{z}}$ from the positron run and $\mathrm{a}$ lineout $(r=0)$ in Fig. 2. The peak accelerating field of 210 $\mathrm{MV} / \mathrm{m}$ is in excellent agreement with results in Ref. [3].

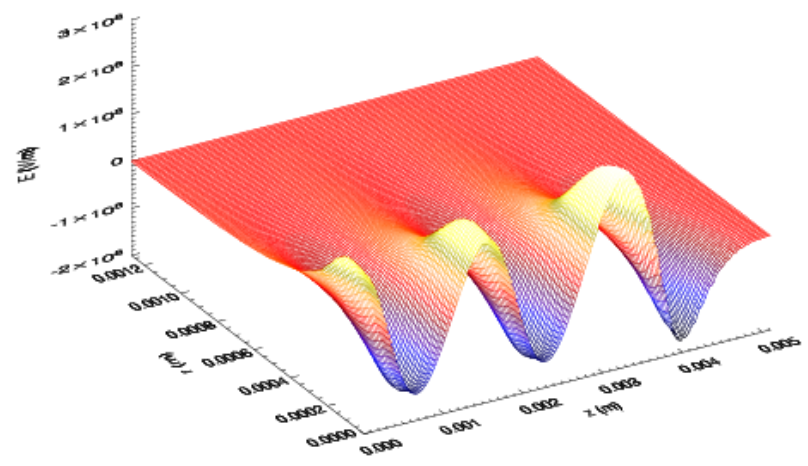

Figure 1: Surface plot of Ez for a positron driver, using parameters on the left side of Table 1. 


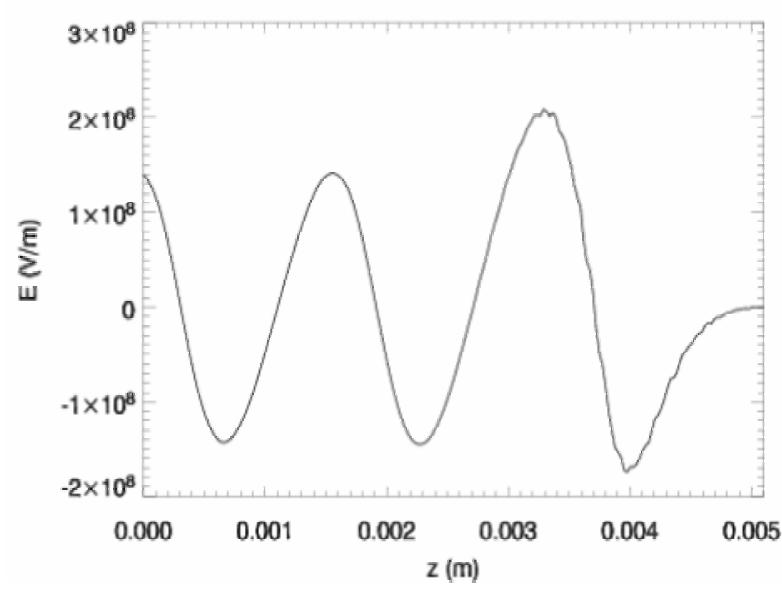

Figure 2: Line-out of $E_{z}$ from Fig. 1, along $r=0$, for a positron driver.

The corresponding results for the electron simulations are shown in Fig.'s 3 and 4, again showing excellent agreement with Ref. [3] in all aspects, except for the details of the narrow field spike, which is an underresolved feature and is not useful for accelerating particles.

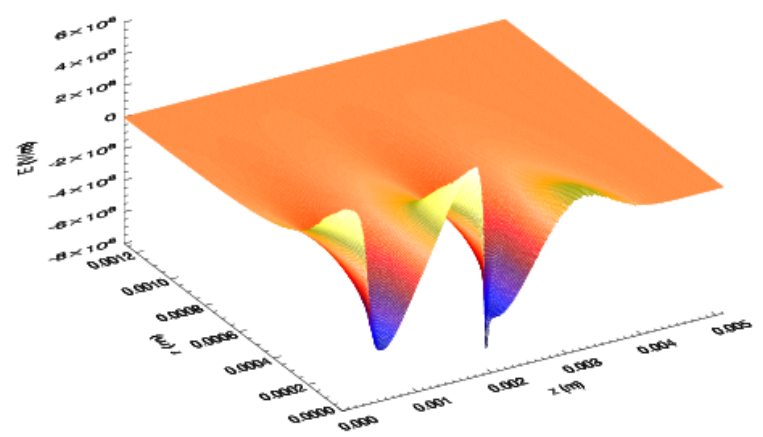

Figure 3: Surface plot of $\mathrm{E}_{\mathrm{z}}$ for an electron driver, using parameters on the left side of Table 1.

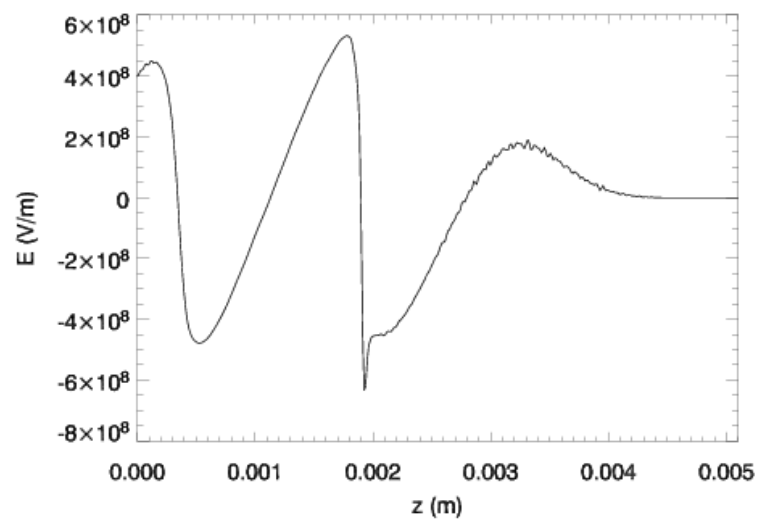

Figure 4: Line-out of $\mathrm{E}_{\mathrm{z}}$ from Fig. 3, along $\mathrm{r}=0$.

\section{E-164x PARAMETERS}

We now consider a new parameter regime, described by the right-hand column of Table 1 , which is relevant to the planned E-164x experiment at SLAC - a follow-on to the presently running E-164 experiment. In choosing the range of densities to simulate, we considered Eq. (1) of Ref. [3] --

$$
E_{z} \sim \frac{n_{b} \sqrt{2 \pi}\left(k_{p}^{2} \sigma_{r}^{2}\right) k_{p} \sigma_{z} \exp \left(-k_{p}^{2} \sigma_{z}^{2} / 2\right)}{\sqrt{n_{o}}\left(1+k_{p}^{2} \sigma_{r}^{2}\right)} \sin \left(k_{p}(z-c t)\right)
$$

which is an approximate expression for the peak electric field in an electron-driven PWFA, derived from linear theory. If $\sigma_{\mathrm{r}}$ and $\sigma_{\mathrm{z}}$ are held fixed, then one can choose an optimal density by maximizing $\mathrm{E}_{\mathrm{z}}$.

In the limit that $\mathrm{k}_{\mathrm{p}} \sigma_{\mathrm{r}}<<1$, one finds the optimal density can be expressed by the equation $\mathrm{k}_{\mathrm{p}} \sigma_{\mathrm{z}}=\operatorname{sqrt}(2)$. For the special case $\mathrm{k}_{\mathrm{p}} \sigma_{\mathrm{r}}=\mathrm{k}_{\mathrm{p}} \sigma_{\mathrm{z}}$, the optimal density is determined by $\mathrm{k}_{\mathrm{p}} \sigma_{\mathrm{z}}=1$. In general, the requirement is

$$
2 k_{p}^{2} \sigma_{r}^{2}=\sqrt{1+8 \sigma_{r}^{2} / \sigma_{z}^{2}} \text {. }
$$

For our parameters, the optimal plasma density is $7 \times 10^{16}$ $\mathrm{cm}^{-3}$, which is comparable to the beam density - putting us into a regime where linear theory is marginally valid.

Figure 5 shows the surface plot of $\mathrm{E}_{\mathrm{z}}$ for a positron driver and a $100 \%$ pre-ionized plasma. The peak value just exceeds $10 \mathrm{GV} / \mathrm{m}$, which is less than the cold wavebreaking field $E_{0} \sim 25 \mathrm{GV} / \mathrm{m}$, consistent with a linear plasma response. Figure 6 shows a color contour plot of the electron number density in the wake.

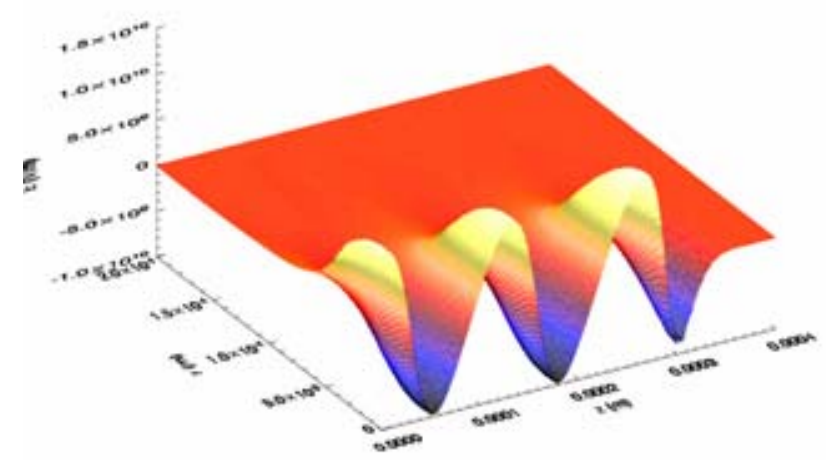

Figure 5: Surface plot of $\mathrm{E}_{\mathrm{z}}$ for a positron driver in a preionized plasma, using parameters on right side of Table 1.

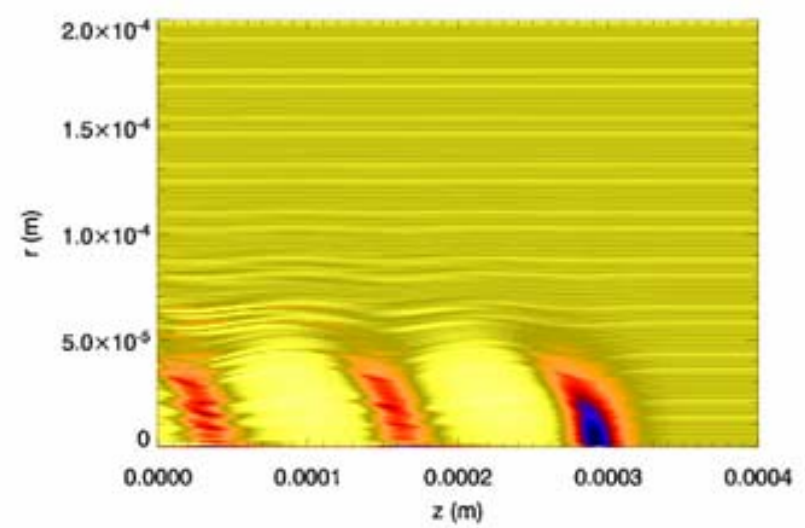

Figure 6: Contour plot of $\mathrm{n}_{\mathrm{e}}$ for same case as in Fig. 5; the high densities create the large wake fields. 
Figure 7 shows the surface plot of $E_{z}$ for a positron driver in a neutral Cs gas. The wakefield is remarkably similar to that of the pre-ionized case, shown above in Fig. 5. Figure 8 shows a color contour plot of the electron number density in the wake - this image differs significantly from Fig. 6, because here the wake electrons are created locally by high electric fields.

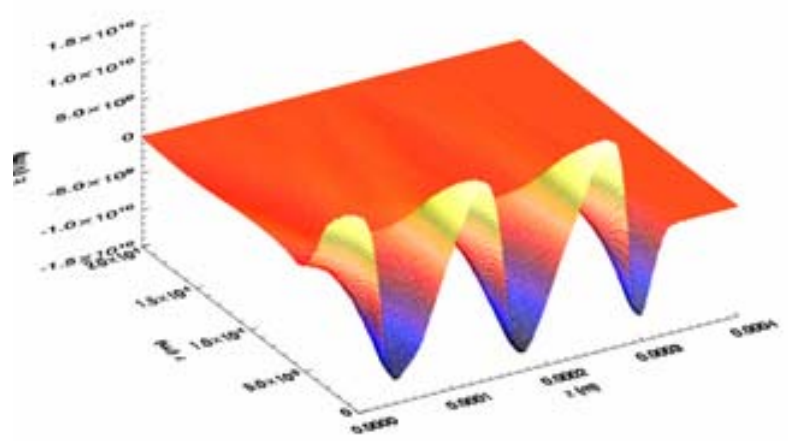

Figure 7: Surface plot of $\mathrm{E}_{\mathrm{z}}$ for a positron driver in neutral $\mathrm{Cs}$, using parameters on right side of Table 1.

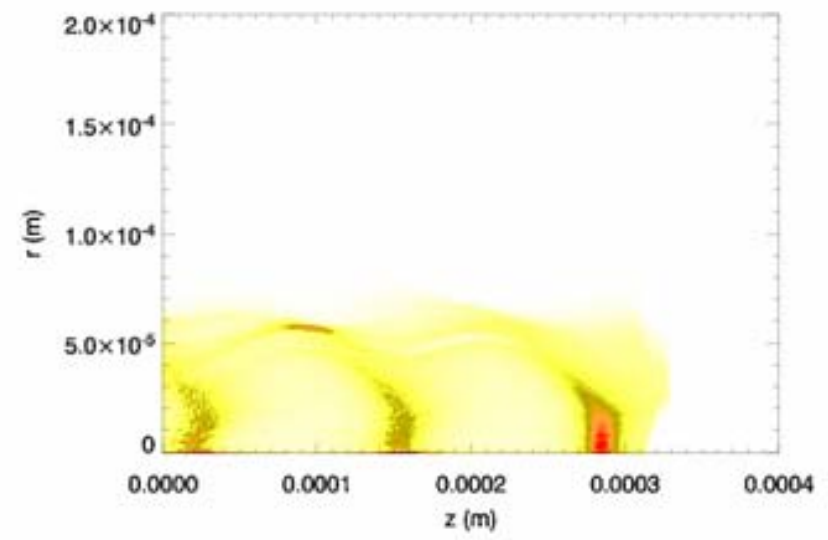

Figure 8: Contour plot of $\mathrm{n}_{\mathrm{e}}$ for same case as in Fig. 7; all wake electrons are generated via tunneling ionization.

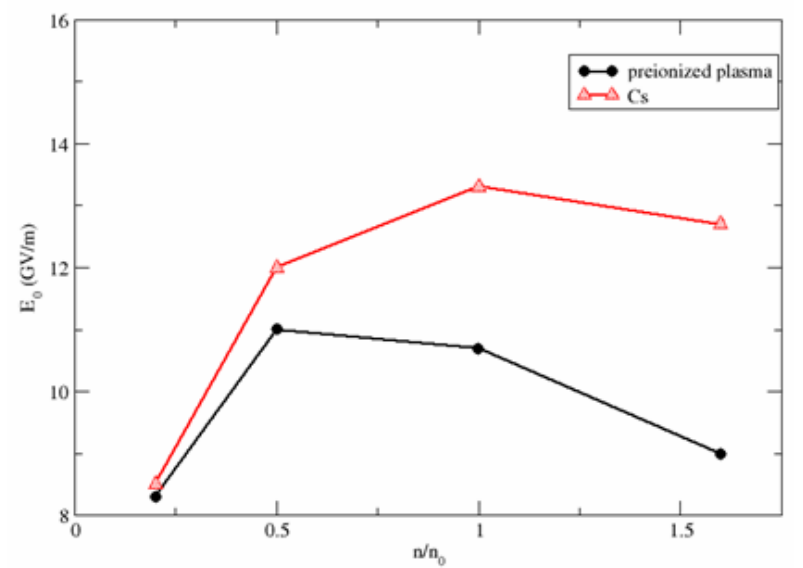

Figure 9: Variation of peak accelerating field with density for positron drivers in pre-ionized plasma \& neutral Cs.

The density of the pre-ionized plasma, and alternatively of the neutral Cs gas, was scanned over a range centered on the prediction from linear theory. Figure 9 presents the peak $E_{z}$ as a function of density, showing that for the neutral Cs case it peaks at slightly higher density and at slightly higher values than for the pre-ionized case.

These results strongly suggest that a high-density positron driver, such as one would find in a "plasma afterburner" scenario, does not require the Cs gas to be pre-ionized. Because it is practically impossible to $100 \%$ pre-ionize a gas, the effects of tunneling ionization would likely disrupt the idealized wake by ionizing much of the background gas. For these reasons, positron-driven PWFA's using a neutral gas like Cs show significant promise.

\section{CONCLUSIONS}

We have presented the first PIC simulations of $\mathrm{e}+$ drivers for a PWFA, including the effects of tunneling ionization. Agreement with previous work for a preionized plasma was shown. For new work, parameters relevant to E-164x were chosen.

For E-164x parameters, the results with TI of neutral Cs are very close to the ideal case of a pre-ionized plasma. In fact, the peak fields in the TI case are slightly higher. Thus, one could likely use neutral Cs gas for a full-scale "plasma afterburner" facility, which would save the expense and complexity of a UV laser system for preionization.

\section{ACKNOWLEDGEMENTS}

The authors thank Richard Busby for his excellent Python scripts, which enable batch mode parameter sweeps and automatic image rendering with OOPIC. We also thank M. Hogan, C. Joshi, T. Katsouleas, K. Marsh, W. Mori and P. Muggli for many helpful discussions.

This work is supported in part by the SciDAC project -Advanced Computing for 21st Century Accelerator Science \& Technology, an initiative of the U.S. Department of Energy, under Contract No. DE-FG0201ER41178. This work is further supported by the U.S. Department of Energy, under Contract No.'s DE-FG0399ER82903, DE-FG03-95ER40926 and DE-AC0376SF00098, and by Tech-X Corporation. This work used resources of the National Energy Research Scientific Computing Center.

\section{REFERENCES}

[1] S. Lee et al., Phys. Rev. S.T. AB 5, 011001 (2002).

[2] D.L. Bruhwiler et al., Phys. Plasmas 10, 2022 (2003).

[3] S. Lee et al., Phys. Rev. E 64, 045501(R) (2001).

[4] D.L. Bruhwiler et al., Phys. Rev. S.T. AB 4, 101302 (2001); J.P. Verboncoeur et al., Comp. Phys. Comm. 87, 199 (1995).

[5] W. Lu et al., Bull. Am. Phys. Soc. 47 (9), 316 (2002).

[6] Esarey et al., IEEE Trans. Plasma Sci. 24, 252 (1996).

[7] P. Chen et al., Phys. Rev. Lett. 54, 693 (1985).

[8] R. Ruth et al., Part. Accel. 17, 171 (1985). 